\title{
Associations between polymorphisms of long non-coding RNA MEG3 and risk of colorectal cancer in Chinese
}

\author{
Xiangming $\mathrm{CaO}^{1, *}$, Shulin Zhuang ${ }^{2, *}$, Youfang $\mathrm{Hu}^{3, *}$, Lei $\mathrm{Xi}^{1}$, Lichun Deng ${ }^{1}$, Huaming \\ Sheng ${ }^{1}$ and Weisheng Shen ${ }^{1}$ \\ ${ }^{1}$ Department of Oncology, The Affiliated Jiangyin Hospital of Southeast University Medical College, Wuxi, China \\ 2 Pukou District Center for Disease Control and Prevention, Nanjing, China \\ ${ }^{3}$ Department of Children's Health, The First Affiliated Hospital of Nanjing Medical University, Nanjing, China \\ * These authors have contributed equally to this work
}

Correspondence to: Weisheng Shen, email: drwsshen@163.com

Keywords: IncRNA, MEG3, colorectal cancer

Received: October 14, $2015 \quad$ Accepted: February 15, $2016 \quad$ Published: February 26, 2016

\section{ABSTRACT}

Maternally expressed gene 3 (MEG3), a long non-coding RNA (IncRNA), is involved in cancer development and metastasis. The objective of the present study was to evaluate whether common single nucleotide polymorphisms (SNPs) in MEG3 could be related with colorectal cancer risk in Chinese. We genotyped six tagSNPs of MEG3 in a colorectal cancer case-control study including 518 cases and 527 control subjects. Multivariate logistic regression analysis was applied to calculate adjusted odds ratios (ORs). We found that MEG3 rs7158663 AA genotype, but not GA genotype, had significant increased colorectal cancer risk, compared with GG genotype (OR = 1.96 and $P=0.006$ for $A A$ versus $G G$, and $O R=1.20$ and $P=0.171$ for $G A$ versus $G G$ ). Further stratified analysis indicated that the increased risk was significantly correlated with individuals with age $\leq \mathbf{6 0}$ and family history of cancer. However, there was no significant association between rs7158663 and colorectal tumor site and stage $(P=$ 0.842 for tumor site, and $P=\mathbf{0 . 6 0 1}$ for tumor stage). These results demonstrate that genetic variants in MEG3 may contribute to the development and risk of colorectal cancer. Further studies are required to confirm these findings.

\section{INTRODUCTION}

Colorectal cancer is the third most commonly diagnosed cancer in the world [1]. According to recent reports, the incidence and mortality of colorectal cancer in China have also been increasing in last two decades [2]. It is well known that colorectal cancer susceptibility is related to multiple environmental factors and genetic alterations, such as genetic mutations or polymorphisms $[3,4]$. Nevertheless, the role of genetic polymorphism and colorectal cancer susceptibility still remains unknown.

Long non-coding RNAs (lncRNAs) are noncoding molecules larger than 200 nucleotides lacking significant protein-coding capacity [5]. It has been reported that lncRNAs are involved in diverse functions in carcinogenesis, including chromatin modification, transcription, and posttranscriptional processing [6]. In addition, previous studies have indicated that polymorphisms in lncRNAs may influence the risk of gastric cancer $[7,8]$. Recent studies have demonstrated that lncRNA maternally expressed gene 3 (MEG3) is abnormal expressed in various human cancers, such as hepatocellular carcinoma [9, 10], bladder cancer [11], glioma [12], and gastric cancer [13]. Moreover, $M E G 3$ overexpression induced cell proliferation and was associated with the development and progression of colorectal cancer [14]. However, little is known about the single nucleotide polymorphisms (SNPs) in MEG3 and colorectal cancer risk.

On the basis of previous findings mentioned above, as well as the influence of SNPs on MEG3, we hypothesis that genetic variants of MEG3 may modify the development of colorectal cancer. The genetic variants of $M E G 3$ may be associated with the expression of $M E G 3$ and consequently influence susceptibility to colorectal cancer. To test the hypothesis, we carried out an association study between tagging SNPs (tagSNPs) in $M E G 3$ and colorectal cancer risk in a hospital-based 
colorectal cancer case-control study comprising 518 patients and 527 control subjects from China.

\section{RESULTS}

The demographic characteristics of participants are described in Table 1. The average age of the patients was 60.0 years old compared with 59.2 years old in controls, which revealed no statistically difference $(P=0.284)$. Furthermore, there was no significant difference in sex distribution $(P=0.972)$ or smoking status $(P=0.292)$. However, the cases were asked to report a significant high rate of family history of cancer than the controls $(P$ $<0.001$ ). Among 518 patients, the number of cases with colon cancer and rectal cancer were $248(47.9 \%)$ and 270 $(52.1 \%)$, respectively. The tumor stage for I, II, III, and IV were 38 (7.3\%), 214 (41.3\%), 179 (34.6\%), and 87 $(16.8 \%)$, respectively.

The primary information of six SNPs in MEG3 is summarized in Table 2. All SNPs in both cases and controls showed a call rate $>97.0 \%$. The genotype frequencies in controls were in line with the HardyWeinberg equilibrium model ( $P=0.712$ for rs3087918, $P=0.930$ for rs $11160608, P=0.812$ for rs7158663, $P=$
0.521 for rs4081134, and $P=0.221$ for rs 10144253).

As shown in Table 2, the allele frequency of rs7158663 exhibited a significant difference between case and control groups $(P=0.007)$. The minor allele frequency (MAF) of rs 7158663 in cases and controls were 0.295 and 0.242 , respectively. However, no significant association with colorectal cancer was identified for other five SNPs $(P=0.493$ for $\mathrm{rs} 3087918, P=0.788$ for $\mathrm{rs} 11160608, P$ $=0.711$ for rs 4081134 , and $P=0.843$ for rs 10144253). In addition, after adjusting for multiple testing using Bonferroni correction, rs7158663 was still significant $(P$ $=0.035$ ).

We further performed a multivariate logistic regression analysis for rs 7158663 by adjusting age, sex, and smoking. As listed in Table 3, rs7158663 AA genotype, but not GA genotype, had a significantly elevated risk of colorectal cancer, compared with GG genotype $(P=$ $0.007)(\mathrm{OR}=1.96$ and $P=0.006$ for $\mathrm{AA}$ versus $\mathrm{GG}$, and $\mathrm{OR}=1.20$ and $P=0.171$ for $\mathrm{GA}$ versus $\mathrm{GG}$ ). For genetic models, rs7158663 was significant between cases and controls in dominant, recessive, and additive models $(P=$ $0.035,0.012$, and 0.007 , respectively).

We next evaluated the stratified association of rs7158663 with colorectal cancer risk by age, sex, family

a

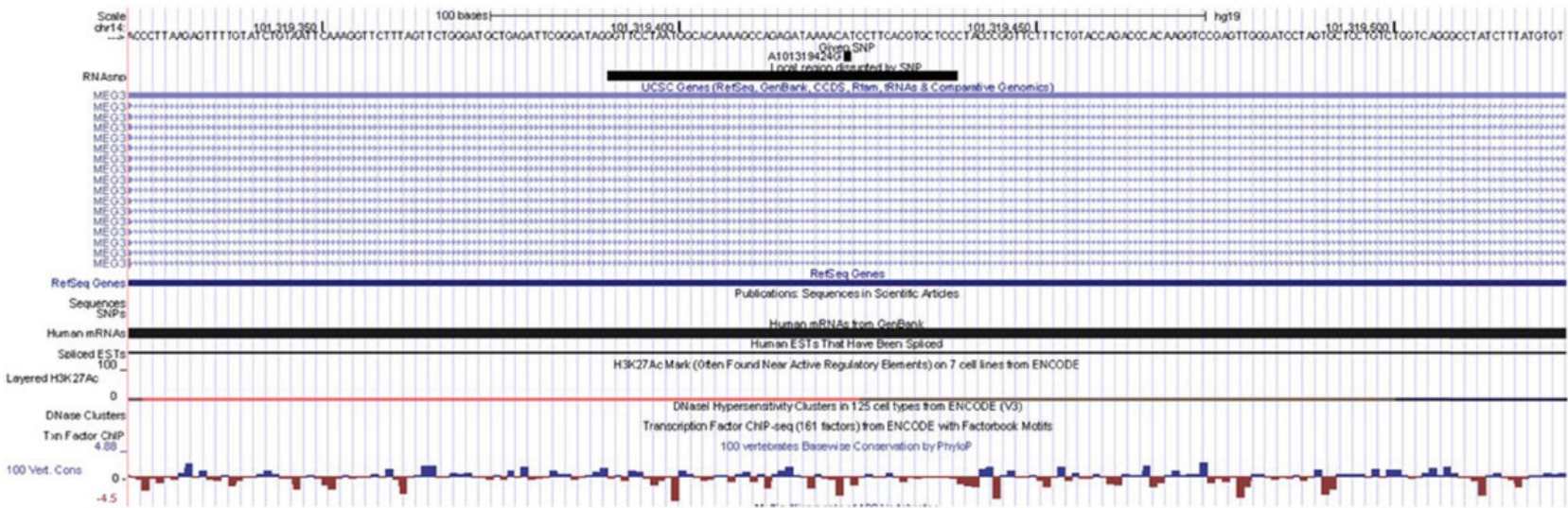

b

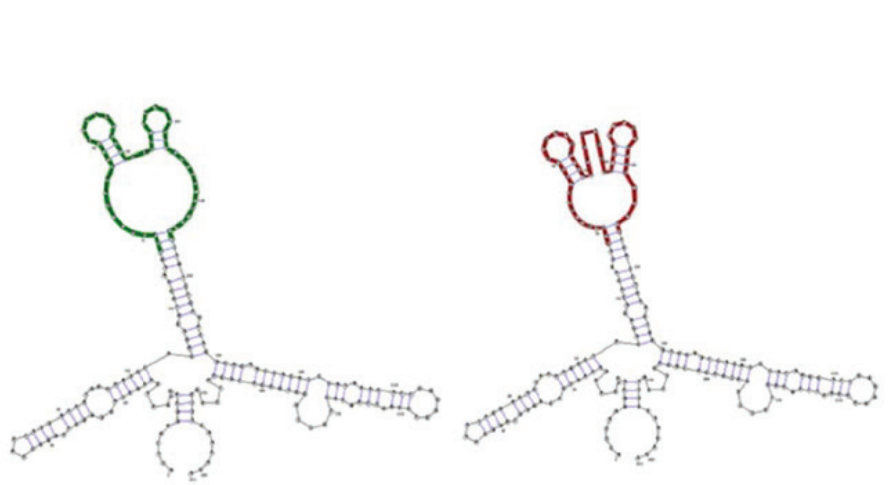

c

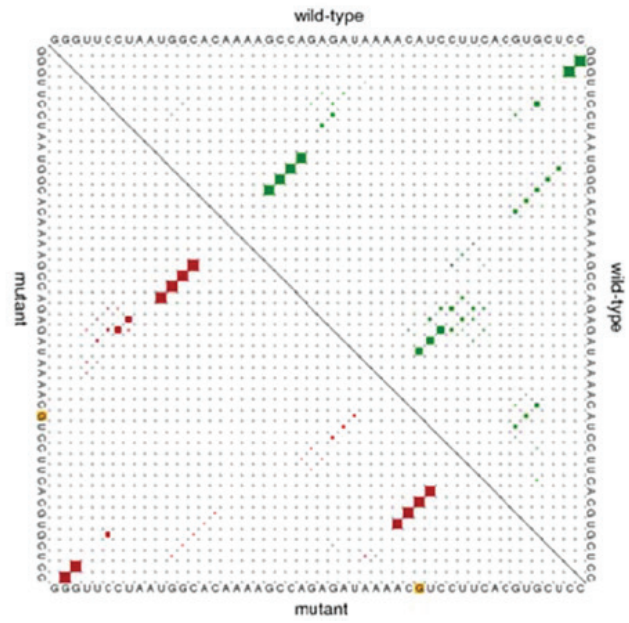

Figure 1: The predicting rs7158663 on MEG3 secondary structure. a. UCSC genome browser view for structure-disruptive rs7158663. b. Minimum free energy structures of rs7158663. c. Dot plot of the global structure. 
Table 1: Distribution of characteristics among cases and controls

\begin{tabular}{|c|c|c|c|}
\hline Variable & Cases $(n=518)$ & Controls $(n=527)$ & $P$ \\
\hline & $N(\%)$ & $N(\%)$ & \\
\hline Age $(\text { mean } \pm \mathrm{SD})^{\mathrm{a}}$ & $60.0 \pm 12.4$ & $59.2 \pm 9.4$ & 0.284 \\
\hline \multicolumn{4}{|l|}{ Gender } \\
\hline Male & $320(61.8)$ & $325(61.7)$ & 0.972 \\
\hline Female & $198(38.2)$ & $202(38.3)$ & \\
\hline \multicolumn{4}{|l|}{ Smoking status } \\
\hline Non-smokers & $347(67.0)$ & $369(70.0)$ & 0.292 \\
\hline Smokers & $171(33.0)$ & $158(30.0)$ & \\
\hline \multicolumn{4}{|c|}{ Family history of cancer } \\
\hline No & $405(78.2)$ & $488(92.6)$ & $<0.001$ \\
\hline Yes & $113(21.8)$ & $39(7.4)$ & \\
\hline \multicolumn{4}{|l|}{ Site } \\
\hline Colon & $248(47.9)$ & & \\
\hline Rectum & $270(52.1)$ & & \\
\hline \multicolumn{4}{|l|}{ Stage } \\
\hline $\mathrm{I}$ & $38(7.3)$ & & \\
\hline II & $214(41.3)$ & & \\
\hline III & $179(34.6)$ & & \\
\hline IV & $87(16.8)$ & & \\
\hline
\end{tabular}

${ }^{\mathrm{a} S D}$, standard deviation

Table 2: Association analyses between SNPs in $M E G 3$ and colorectal cancer risk

\begin{tabular}{|l|l|l|l|l|l|l|l|l|}
\hline SNP & Position & MAF in casea & MAF in control & Call rate & $\begin{array}{l}\text { HWE } \\
\text { in case }\end{array}$ & $\begin{array}{l}\text { HWE in } \\
\text { control }\end{array}$ & OR $(\mathbf{9 5 \% C I ) b}$ & $\boldsymbol{P}^{\mathbf{b}}$ \\
\hline rs3087918 & 101297963 & 0.367 & 0.381 & $100.0 \%$ & 0.344 & 0.712 & $0.94(0.79-1.12)$ & 0.493 \\
\hline rs11160608 & 101313093 & 0.433 & 0.436 & $100.0 \%$ & 1.000 & 0.930 & $0.98(0.82-1.16)$ & 0.788 \\
\hline rs7158663 & 101319424 & 0.295 & 0.242 & $98.9 \%$ & 0.138 & 0.812 & $1.31(1.08-1.59)$ & 0.007 \\
\hline rs4081134 & 101321788 & 0.227 & 0.219 & $97.8 \%$ & 0.314 & 0.521 & $1.04(0.84-1.29)$ & 0.711 \\
\hline rs10144253 & 101325962 & 0.470 & 0.472 & $99.8 \%$ & 0.659 & 0.221 & $0.98(0.82-1.17)$ & 0.843 \\
\hline
\end{tabular}

${ }^{a}$ Minor allele frequency.

${ }^{\mathrm{b}}$ Additive model adjusted for age, sex, and smoking status.

Table 3: Genotype frequencies of $M E G 3$ rs7158663 among cases and controls and their association with colorectal cancer risk

\begin{tabular}{|l|l|l|l|l|}
\hline Genotype & Cases $(\boldsymbol{n}=\mathbf{5 1 6})$ & Controls $(\boldsymbol{n}=\mathbf{5 1 7})$ & OR $(\mathbf{9 5 \% C I ) a}$ & $\boldsymbol{P}^{\text {a }}$ \\
\hline & $\boldsymbol{N}(\mathbf{\%})$ & $\boldsymbol{N}(\mathbf{\%})$ & & \\
\hline GG & $264(51.2)$ & $298(57.6)$ & 1.00 & \\
\hline GA & $200(38.7)$ & $188(36.4)$ & $1.20(0.92-1.56)$ & 0.171 \\
\hline AA & $52(10.1)$ & $31(6.0)$ & $1.96(1.22-3.17)$ & 0.006 \\
\hline Dominant & & & $1.31(1.02-1.67)$ & 0.035 \\
\hline Recessive & & & $1.82(1.14-2.91)$ & 0.012 \\
\hline Additive & & & $1.31(1.08-1.59)$ & 0.007 \\
\hline
\end{tabular}

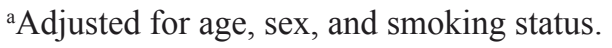


Table 4: Stratified analyses for $M E G 3$ rs7158663 genotypes in cases and controls

\begin{tabular}{|l|l|l|l|l|}
\hline Variable & GG & GA/AA & OR $(\mathbf{9 5} \% \mathbf{C I})^{\mathbf{a}}$ & $\boldsymbol{P}^{\mathbf{a}}$ \\
\hline & $\boldsymbol{N}($ case/control) & $\boldsymbol{N}($ case/control) & & \\
\hline Age (y), median & & & & \\
\hline$\leq 60$ & $104 / 158$ & $124 / 111$ & $1.71(1.20-2.44)$ & 0.003 \\
\hline$>60$ & $160 / 140$ & $128 / 108$ & $1.04(0.74-1.46)$ & 0.844 \\
\hline Gender & & & & \\
\hline Male & $167 / 177$ & $152 / 142$ & $1.15(0.84-1.57)$ & 0.396 \\
\hline Female & $97 / 121$ & $100 / 77$ & $1.62(0.98-2.34)$ & 0.076 \\
\hline Smoking status & & & & \\
\hline Non-smokers & $168 / 207$ & $178 / 155$ & $1.42(0.96-1.87)$ & 0.062 \\
\hline Smokers & $96 / 91$ & $74 / 64$ & $1.10(0.70-1.71)$ & 0.679 \\
\hline Family history of cancer & & & & \\
\hline No & $204 / 268$ & $200 / 210$ & $1.25(0.96-1.63)$ & 0.099 \\
\hline Yes & $60 / 30$ & $52 / 9$ & $2.98(1.28-6.92)$ & 0.011 \\
\hline
\end{tabular}

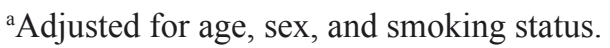

Table 5: Association analyses between $M E G 3$ rs7158663 genotypes and clinicalpathologic characteristics of cases

\begin{tabular}{|l|l|l|l|l|}
\hline Variable & GG & GA/AA & OR $(\mathbf{9 5} \% \mathbf{C I})^{\mathbf{a}}$ & $\boldsymbol{P}^{\mathbf{a}}$ \\
\hline & $\mathbf{N}(\mathbf{\%})$ & $\boldsymbol{N}(\mathbf{\%})$ & & \\
\hline Site & & & & \\
\hline Colon & $126(24.4)$ & $122(23.6)$ & 1.00 & \\
\hline Rectum & $138(26.7)$ & $130(25.2)$ & $1.04(0.73-1.46)$ & 0.842 \\
\hline Stage & & & & \\
\hline I/II & $126(24.4)$ & $125(24.2)$ & 1.00 & \\
\hline III/IV & $138(26.7)$ & $127(24.6)$ & $1.10(0.78-1.55)$ & 0.601 \\
\hline
\end{tabular}

${ }^{a}$ Adjusted for age, sex, and smoking status.

history of cancer, and smoking habits (Table 4). We observed that the GA/AA genotype hadan increased risk of colorectal cancer $(\mathrm{OR}=1.31, P=0.035)$. This increased effect was also more evident in subgroups of age $\leq 60$ (OR $=1.71, P=0.003)$ and patients with family history $(\mathrm{OR}$ $=1.25, P=0.011)$. Nevertheless, no significant evidence was found for interaction between rs 7158663 and these two factors.

We evaluated the correlations between rs 7158663 and clinical features of colorectal cancer, including tumor site and stage. As shown in Table 5, no significant relationship was observed $(P=0.842$ for tumor site, and $P$ $=0.601$ for tumor stage $)$.

We peformed an in silico analysis of rs 7158663 on MEG3 by RNAsnp. As shown in Figure 1, the RNAsnp predicted that rs $7158663 \mathrm{G}-\mathrm{A}$ allele substitution leaded to a minimum free energy (MFE) change from -53.30 $\mathrm{kcal} / \mathrm{mol}$ to $-55.20 \mathrm{kcal} / \mathrm{mol}$. Moreover, the base pair probabilities were different between rs $7158663 \mathrm{G}$ allele and $A$ allele.

\section{DISCUSSION}

We investigated the association of tagSNPs of $M E G 3$ with colorectal cancer risk in a population of Chinese. We demonstrated, for the first time, that rs7158663 in MEG3 had a strong association with increased risk of colorectal cancer. In addition, the elevated risk was greater for subjects with age $\leq 60$ and family history of cancer. These results suggested that rs7158663 may be a susceptibility SNP for colorectal cancer in Chinese populations.

Given $M E G 3$ is a lncRNA that plays critical roles in tumor cellular proliferation, migration and invasion [13, $15,16]$, SNPs in $M E G 3$ may affect cell phenotypes and cause the risk of developing cancer. $M E G 3$ is located on chromosome $14 \mathrm{q} 32$, a region proposed to contain putative tumor suppressors [17]. Although the regulation of $M E G 3$ and its precise mechanism of action in cancer are still well known, emerging evidence strongly demonstrates that $M E G 3$ functions as a novel lncRNA tumor suppressor [18]. In additional to colorectal normal tissue, MEG3 is highly expressed in brain, lung, and liver [19]. 
Interestingly, we found that $M E G 3$ rs7158663 is correlated to significantly increased risk of colorectal cancer, which may be explained by the finds from Yin et al. [14]. They found that $M E G 3$ predicts a poor prognosis of colorectal cancer by influencing cell proliferation.

Until now, some studies have indicated that SNPs in lncRNAs could potentially impact various biological processes by influencing biological pathways. For example, Yang et al. first revealed the relationship between IncRNA H19 and gastric cancer; they found an important role for $\mathrm{H} 19$ variants in gastric cancer carcinogenesis [8]. Zhang et al. identified an allelic regulation of rs 920778 on IncRNA HOTAIR expression, as well as associated with the development and progression of esophageal squamous cell carcinoma [20]. The novel function of rs920778 on HOTAIR was further confirmed in other cancer, including colorectal cancer [21], gastric cancer [22], and breast cancer [23]. The RNAsnp prediction revealed that rs7158663 in $M E G 3$ changed the folding structures of $M E G 3$. Therefore, we speculate that rs 7158663 could be a regulatory SNP, which regulate expression of $M E G 3$ and contribute to genetic susceptibility of colorectal cancer.

Several limitations of the present study should be mentioned. Firstly, these findings were based on genetic analysis of a single gene with a relatively small size of colorectal cancer patients, which need to be validated using an independent prospective clinical study. Secondly, our study was based on a hospital-based study; therefore, potentially important sources of selection bias may exist. Thirdly, diet is a major determinant of risk for colorectal cancer [24]; however, our study had incomplete information on dietary consumption. Further studies are needed to determine the association between dietary information and genetic variants in $M E G 3$.

In summary, we identified a genetic susceptibility SNP rs7158663 for colorectal cancer in Chinese. The SNP rs7158663 in MEG3 played a vital role in colorectal carcinogenesis. These data suggest that genetic variants in $M E G 3$ may serve as potential risk factor and targets for colorectal cancer therapy in the further.

\section{MATERIALS AND METHODS}

\section{Study subjects}

This study consisted of 518 colorectal cancer cases and 527 control subjects from the hospitals of Southeast University Medical College. All cases were patients newly diagnosed with histologically confirmed colorectal adenocarcinomas who were admitted to the hospitals. No patient had received radiotherapy or chemotherapy. Controls were frequency-matched with cases on age and sex who were recruited at the same time period. All controls were unrelated ethnic Han Chinese and these subjects had no history of cancer. All participants provided informed consent after the interview. This research protocol was approved by the Institutional Review Board of Southeast University Medical College.

\section{SNPs selection}

We selected the tagSNPs of MEG3 with the MAF > 0.05 in Han Chinese from the 1000 Genome Projects. As a result, six tagSNPs were selected using a pairwise Tagger method with $r^{2}>0.8$ to capture other SNPs, rs 10144253 .

\section{Genotyping}

Genomic DNA was extracted from peripheral blood using the TIANamp Blood DNA kit (Tiangen, China). Genotyping was performed by TaqMan SNP genotyping assay. Real-time TaqMan PCR and genotyping were conducted on an ABI 7500 real-time PCR System (Applied Biosystems, USA). The results of allelic discrimination were analyzed using SDS 2.4 software. For quality control, we included two negative controls (water) and two duplicates in each 96-well plate. Furthermore, about $3 \%$ of selected samples were repeated genotyping to confirm the results in a blind fashion.

\section{Statistical analysis}

Tests for the Hardy-Weinberg equilibrium in cases and controls were performed by good-of-fit $\chi^{2}$ test. Student's t-test or $\chi^{2}$ test was used to assess the significance of any differences in frequency distributions of demographic variables and genotypes among cases and controls. We estimated the association between genotypes and colorectal cancer risk by odds ratios (ORs) and $95 \%$ confidence intervals (CIs) using the logistic regression. The ORs and $95 \%$ CIs were further adjusted by for age, sex, and smoking habits. All analyses were two-sided and $P<0.05$ was considered significant. All statistical calculations were conducted with SPSS 13.0 software (SPSS Inc., Chicago, IL, USA).

\section{CONFLICTS OF INTERESTS}

We declared no conflicts of interests in this study.

\section{REFERENCES}

1. Ferlay J, Soerjomataram I, Dikshit R, Eser S, Mathers C, Rebelo M, Parkin DM, Forman D and Bray F. Cancer incidence and mortality worldwide: sources, methods and major patterns in GLOBOCAN 2012. International journal of cancer. 2015; 136:E359-386. 
2. Chen W, Zheng R, Zhang S, Zhao P, Li G, Wu L and He J. Report of incidence and mortality in China cancer registries, 2009. Chinese journal of cancer research [article in Chineese]. 2013; 2 5:10-21.

3. Ma X, Zhang B and Zheng W. Genetic variants associated with colorectal cancer risk: comprehensive research synopsis, meta-analysis, and epidemiological evidence. Gut. 2014; 63:326-336.

4. Tenesa A and Dunlop MG. New insights into the aetiology of colorectal cancer from genome-wide association studies. Nature reviews Genetics. 2009; 10:353-358.

5. Costa FF. Non-coding RNAs: Meet thy masters. BioEssays. 2010; 32:599-608.

6. Wilusz JE, Sunwoo H and Spector DL. Long noncoding RNAs: functional surprises from the RNA world. Genes \& development. 2009; 23:1494-1504.

7. Du M, Wang W, Jin H, Wang Q, Ge Y, Lu J, Ma G, Chu $\mathrm{H}$, Tong N, Zhu H, Wang M, Qiang F and Zhang Z. The association analysis of lncRNA HOTAIR genetic variants and gastric cancer risk in a Chinese population. Oncotarget. 2015; 6:31255-31262. doi: 10.18632/oncotarget.5158.

8. Yang C, Tang R, Ma X, Wang Y, Luo D, Xu Z, Zhu Y and Yang L. Tag SNPs in long non-coding RNA H19 contribute to susceptibility to gastric cancer in the Chinese Han population. Oncotarget. 2015; 6:15311-15320. doi: 10.18632/oncotarget.3840.

9. Braconi C, Kogure T, Valeri N, Huang N, Nuovo G, Costinean S, Negrini M, Miotto E, Croce CM and Patel T. microRNA-29 can regulate expression of the long non-coding RNA gene MEG3 in hepatocellular cancer. Oncogene. 2011; 30:4750-4756.

10. Zhuo H, Tang J, Lin Z, Jiang R, Zhang X, Ji J, Wang P and Sun B. The aberrant expression of MEG3 regulated by UHRF1 predicts the prognosis of hepatocellular carcinoma. Molecular carcinogenesis. 2016; 55:209-19. doi: 10.1002/ mc. 22270 .

11. Ying L, Huang Y, Chen H, Wang Y, Xia L, Chen Y, Liu $\mathrm{Y}$ and Qiu F. Downregulated MEG3 activates autophagy and increases cell proliferation in bladder cancer. Molecular bioSystems. 2013; 9:407-411.

12. Wang $P$, Ren $Z$ and Sun P. Overexpression of the long non-coding RNA MEG3 impairs in vitro glioma cell proliferation. Journal of cellular biochemistry. 2012; 113:1868-1874.

13. Yan J, Guo X, Xia J, Shan T, Gu C, Liang Z, Zhao W and Jin S. MiR-148a regulates MEG3 in gastric cancer by targeting DNA methyltransferase 1. Medical oncology. 2014; $31: 879$.
14. Yin DD, Liu ZJ, Zhang E, Kong R, Zhang ZH and Guo RH. Decreased expression of long noncoding RNA MEG3 affects cell proliferation and predicts a poor prognosis in patients with colorectal cancer. Tumour biology. 2015; 36:4851-4859.

15. Lu KH, Li W, Liu XH, Sun M, Zhang ML, Wu WQ, Xie WP and Hou YY. Long non-coding RNA MEG3 inhibits NSCLC cells proliferation and induces apoptosis by affecting p53 expression. BMC cancer. 2013; 13:461.

16. Balik V, Srovnal J, Sulla I, Kalita O, Foltanova T, Vaverka M, Hrabalek L and Hajduch M. MEG3: a novel long noncoding potentially tumour-suppressing RNA in meningiomas. Journal of neuro-oncology. 2013; 112:1-8.

17. Bando T, Kato Y, Ihara Y, Yamagishi F, Tsukada K and Isobe M. Loss of heterozygosity of $14 \mathrm{q} 32$ in colorectal carcinoma. Cancer genetics and cytogenetics. 1999; 111:161-165.

18. Zhou Y, Zhang X and Klibanski A. MEG3 noncoding RNA: a tumor suppressor. Journal of molecular endocrinology. 2012; 48:R45-53.

19. Zhang X, Zhou Y, Mehta KR, Danila DC, Scolavino S, Johnson SR and Klibanski A. A pituitary-derived MEG3 isoform functions as a growth suppressor in tumor cells. The Journal of clinical endocrinology and metabolism. 2003; 88:5119-5126.

20. Zhang X, Zhou L, Fu G, Sun F, Shi J, Wei J, Lu C, Zhou C, Yuan Q and Yang M. The identification of an ESCC susceptibility SNP rs920778 that regulates the expression of IncRNA HOTAIR via a novel intronic enhancer. Carcinogenesis. 2014; 35:2062-2067.

21. Xue Y, Gu D, Ma G, Zhu L, Hua Q, Chu H, Tong N, Chen $\mathrm{J}$, Zhang $\mathrm{Z}$ and Wang $\mathrm{M}$. Genetic variants in lncRNA HOTAIR are associated with risk of colorectal cancer. Mutagenesis. 2015; 30:303-310.

22. Bayram S, Ulger Y, Sumbul AT, Kaya BY, Rencuzogullari A, Genc A, Sevgiler Y, Bozkurt O and Rencuzogullari E. A functional HOTAIR rs920778 polymorphism does not contributes to gastric cancer in a Turkish population: a case-control study. Familial cancer. 2015; 14:561-7. doi: 10.1007/s10689-015-9813-0.

23. Bayram S, Sumbul AT, Batmaci CY and Genc A. Effect of HOTAIR rs920778 polymorphism on breast cancer susceptibility and clinicopathologic features in a Turkish population. Tumour biology. 2015; 36:3863-3870.

24. Singh PN and Fraser GE. Dietary risk factors for colon cancer in a low-risk population. American journal of epidemiology. 1998; 148:761-774. 